\title{
Research Paper: Comparing APACHE II, APACHE IV, SAPS II, and SOFA Predictive Power in Poisoned Patients Admitted to the Intensive Care Unit
}

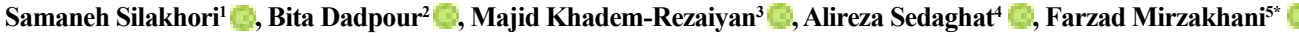 \\ 1. Department of Pharmacodynamics and Toxicology, School of Pharmacy, Mashhad University of Medical Sciences, Mashhad, Iran. \\ 2. Department of Medical Toxicology, School of Medicine, Imam Reza Hospital, Mashhad University of Medical Sciences, Mashhad, Iran. \\ 3. Clinical Research Development Unit, Faculty of Medicine, Mashhad University of Medical Sciences, Mashhad, Iran. \\ 4. Lung Disease Research Center, Faculty of Medicine, Mashhad University of Medical Science, Mashhad, Iran. \\ 5. Department of Health Information Management, School of Health Management and Information Sciences, Iran University of Medical Sciences, \\ Tehran, Iran.
}

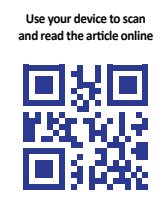

Citation: Silakhori S, Dadpour B, Khadem-Rezaiyan M, Sedaghat A, Mirzakhani F. Comparing APACHE II, APACHE IV, SAPS II, and SOFA Predictive Power in Poisoned Patients Admitted to the Intensive Care Unit. International Journal of Medical Toxicology and Forensic Medicine. 2020; 10(2):28814. https://doi.org/10.32598/ijmtfm.v10i2.28814

https://doi.org/10.32598/ijmtfm.v10i2.28814

\section{(A) 03}

Article info:

Received: 27 Jan 2020

First Revision: 05 Feb 2020

Accepted: 10 Mar 2020

Published: 20 Jun 2020

\section{Keywords:}

Critical jare, Prognostic models, Acute Physiology And Chronic Health Evaluation (APACHE), Simplified acute physiology score, Organ dysfunction scores

\section{A B S T R A C T}

Background: This study aimed to assess the performance of the Acute Physiology and Chronic Health Evaluation (APACHE) II, APACHE IV, Simplified Acute Physiologic Score (SAPS) II, and Sequential Organ Failure Assessment (SOFA) scores in predicting mortality rate in poisoning patients admitted to an intensive care unit (ICU).

Methods: This cross-sectional study was performed on all admitted patients in the poisoning ICU of Imam Reza Hospital, Mashhad City, Iran. All patients were evaluated for three consecutive days since admission time and then every two days until discharge from ICU or death. The scoring systems mentioned above were calculated and analyzed by MedCalc statistical software version 18.9.1 and SPSS version 16.

Results: Overall, 150 patients were studied, out of whom 67\% (101) were male. Their mean \pm SD age was $41.6 \pm 18.9$ years. In their whole hospitalization period, APACHE II (79.5\%), SAPS II (78.7\%), APACHE IV (78.4\%), and SOFA (72.9\%) were the most precise measures. On the first day of admission APACHE II (77.4\%), on the second day, APACHE II $(83.1 \%)$, on the third day, APACHE II $(90.7 \%)$, and on the fifth day, SOFA $(81.6 \%)$ were the most precise measures.

Conclusion: All four systems have acceptable discriminatory power for poisoned patients. However, it seems that APACHE II can be used for mortality prediction, especially in the early days of admission.

\section{* Corresponding Author: \\ Farzad Mirzakhani, PhD.}

Address: Department of Health Information Management, School of Health Management and Information Sciences, Iran University of Medical

Sciences, Tehran, Iran.

Tel: +98 (918) 4341407

E-mail: mirzakhani.f@iums.ac.ir 


\section{Introduction}

oisoning is a significant global public health problem that is associated with $\mathbf{P}$ a considerable number of preventable mortalities and morbidities [1]. Every year many people around the world are poisoned for various causes, such as accidental, intentional, or occupational substance abuse and admitted to emergency units. Only in the United States, more than 2.1 million people sought help by telephone from poison centers in 2016 [2]. On the other side of the world, the situation is not very different: poisoning in Iran also has a high prevalence; approximately $15 \%-20 \%$ of those referring to emergency departments are poisoned patients [3]. Poisoning is also the most common cause of hospitalization, and drug poisoning is the second leading cause of death in hospitalized patients in Iran [4].

The prediction of prognosis for the admitted poisoned patient is essential for different reasons. On the one hand, the choice of treatment method and the level of patient care can be strongly influenced; on the other hand, there is the anxiety of patients' companions waiting for a response from the medical team concerning the probable outcome of their patient. Eventually, with the existence of an objective risk assessment system (i.e., a prognosis prediction system or severity of disease scoring system), the results of the treatment of poisoned patients in different centers can be compared and analyzed.

Today, various prognosis prediction systems are used to determine the likelihood of death of patients admitted to Intensive Care Units (ICUs). These systems, which work based on the severity of the disease, have become increasingly common in medical care in recent decades and can be used for resource allocation, clinical decision-making, and assessment of the quality of care, especially for hospitalized patients in the ICUs $[5,6]$. Such systems are Acute Physiology and Chronic Health Evaluation (APACHE) version II and IV, Simplified Acute Physiologic Score (SAPS II), and Sequential Organ Failure Assessment (SOFA). Each of these four criteria measures specific items. SOFA examines 11 items and is the simplest one in terms of the number of items. The level of consciousness, the need for a ventilator, oxygen saturation, and the oxygen requirement are common items among all of these four systems. On the other hand, APACHE IV uses the most items.

As mentioned, each scoring system has its pros and cons. To our knowledge, no studies have ever compared the performance of prognostic prediction systems among poisoned patients in the ICU. Thus, the purpose of this study is to assess the performance of the APACHE II, APACHE IV, SAPS II, and SOFA scores for their prediction of mortality in poisoned patients during their ICU admission.

\section{Materials and Methods}

This cross-sectional study was performed on all admitted patients to the poisoning ICU of Imam Reza Hospital, Mashhad City, Iran in one Persian calendar year starting from March 20, 2016. This 1500 bed tertiary hospital in the northeast of Iran is the only academic center that has the Toxicology Department.

All patients were evaluated for three consecutive days since their admission time and then every two days until discharge from ICU or death. Demographic (age, gender, history of severe organ failure or immunocompromised, chronic diseases, mechanism of poisoning, length of stay [LOS] in ICU/ward), clinical (acute renal failure, temperature, mean arterial pressure, $\mathrm{pH}$, heart rate/pulse, respiratory rate, systolic blood pressure, mechanical ventilation or continuous positive airway pressure, administration of vasopressors) and paraclinical data (sodium, potassium, creatinine, bilirubin, albumin, blood sugar level, hematocrit, white blood cell count, Glasgow Coma Scale [GCS], $\mathrm{FiO}_{2}, \mathrm{PaO}_{2}, \mathrm{PCO}_{2}$, urine output, blood urea nitrogen, bicarbonate, platelets), which were required to calculate the risk scores, were obtained from patients' files and hospital information system. Mortality was defined as death within the hospitalization period.

The obtained data were analyzed in MedCalc statistical software V. 18.9.1 (MedCalc Software bvba, Ostend, Belgium; http://www.medcalc.org; 2018) and SPSS V. 16. Descriptive (Mean $\pm \mathrm{SD}$, median, range, frequency, percentage) and inferential statistics (Chi-squared test, Student t-test) were used. The discriminating capacity of scoring systems over in-hospital mortality prediction was measured via the Receiver Operating Characteristic (ROC) curves, and the De Long test was conducted to compare the differences between the Area Under Curve (AUC) pairs.

Univariate logistic regression models assessed the probable association of each scoring system with in-hospital mortality. The performance and goodness of fit were evaluated by the Hosmer-Lemeshow test statistic, the Nagelkerke pseudo-R2, the Cox and Snell R2, and the overall correct classification index. All tests were twotailed, and $\mathrm{P}<0.05$ was considered statistically significant. 


\section{Results}

During the one year study, 150 patients were investigated, of whom $67 \%$ (101) were male. Their mean \pm SD age was $41.6 \pm 18.9$ years (median $=37$, range $=14-98$ years). The mean hospitalization period in ICU was $10.3 \pm 13.5$ days (median $=5$, range $=1-75$ days). Totally, 17 patients (11\%) died.

As Table 1 shows, the deceased patients were significantly older, stayed longer in hospital and ICU, and had lower GCS. However, gender, mechanical ventilation, and diagnosis were not different between survived and deceased groups. Moreover, all four predicting tools had a significantly higher score in deceased patients.
During the hospitalization period, APACHE II (79.5\%), SAPS II (78.7\%), APACHE IV (78.4\%), and SOFA $(72.9 \%)$ were the most precise measures (Figure 1). In the first day of admission: APACHE II (77.4\%) (compared to APACHE IV [73.9\%, P=0.56], SAPS II [76.4\%, $\mathrm{P}=0.82]$, and SOFA [72.1\%, $\mathrm{P}=0.13]$ ); in the second day: APACHE II (83.1\%) (compared to APACHE IV [74.9\%, $\mathrm{P}=0.16]$, SAPS II [75.5\%, $\mathrm{P}=0.16]$, and SOFA $[67.8 \%, \mathrm{P}=0.01])$; in the third day: APACHE II $(90.7 \%)$ (compared to APACHE IV [85.1\%, $\mathrm{P}=0.20]$, SAPS II [86.1\%, $\mathrm{P}=0.11]$, and SOFA [72.2\%, $\mathrm{P}<0.001])$; and in the fifth day: SOFA (81.6\%) (compared to APACHE II [76.3\%, $\mathrm{P}=0.32$ ], APACHE IV [74.9\%, $\mathrm{P}=0.30]$, and SAPS II $[79.9 \%, \mathrm{P}=0.75]$ ) were the most precise measures for predicting the prognosis of patients (Figure 2).

Table 1. Comparison of some of the main demographic and clinical variables along with four prediction tools in survived and deceased patients

\begin{tabular}{|c|c|c|c|c|c|}
\hline \multicolumn{2}{|c|}{ Variables } & $\begin{array}{c}\text { Total } \\
(n=150)\end{array}$ & $\begin{array}{c}\text { Survived } \\
(n=133)\end{array}$ & $\begin{array}{c}\text { Deceased } \\
(n=17)\end{array}$ & $\mathbf{P}$ \\
\hline \multicolumn{2}{|c|}{ Age (y) } & $41.62 \pm 18.98$ & $39.26 \pm 17.55$ & $59.82 \pm 20.21$ & 0.001 \\
\hline \multicolumn{2}{|c|}{ LOS in ICU (d) } & $10.38 \pm 13.59$ & $8.86 \pm 11.32$ & $22.23 \pm 22.26$ & 0.026 \\
\hline \multicolumn{2}{|c|}{ LOS in ward (d) } & $0.36 \pm 1.45$ & $0.20 \pm 0.82$ & $1.64 \pm 3.48$ & 0.107 \\
\hline \multicolumn{2}{|c|}{ GCS } & $6.26 \pm 4.75$ & $6.77 \pm 4.95$ & $3.18 \pm 0.63$ & 0.001 \\
\hline \multicolumn{2}{|c|}{ SOFA } & $5.38 \pm 2.99$ & $5.08 \pm 2.91$ & $7.70 \pm 2.71$ & 0.001 \\
\hline \multicolumn{2}{|c|}{ SAPS II } & $30.24 \pm 16.80$ & $28.12 \pm 15.83$ & $46.82 \pm 15.24$ & 0.001 \\
\hline \multicolumn{2}{|c|}{ APACHE II } & $14.45 \pm 7.73$ & $13.48 \pm 7.28$ & $22.00 \pm 7.11$ & 0.001 \\
\hline \multicolumn{2}{|c|}{ APACHE IV } & $47.04 \pm 22.95$ & $44.81 \pm 22.15$ & $64.52 \pm 22.13$ & 0.001 \\
\hline \multirow{2}{*}{ Gender } & Male & $101(67.3)$ & $89(88.1)$ & 12 (11.9) & \multirow{2}{*}{0.761} \\
\hline & Female & $49(32.7)$ & $44(89.8)$ & $5(10.2)$ & \\
\hline \multirow[b]{2}{*}{ Mechanical Ventilation } & No & $79(52.7)$ & 72 (91.1) & $7(8.9)$ & \multirow[b]{2}{*}{0.314} \\
\hline & Yes & $71(47.3)$ & 61 (85.9) & $10(14.1)$ & \\
\hline \multirow{6}{*}{ Diagnosis (poisoning) } & Phosphide & $17(11.3)$ & $17(100)$ & $0(0)$ & \multirow{6}{*}{ NA } \\
\hline & Organophosphate & $9(6.0)$ & $9(100)$ & $0(0)$ & \\
\hline & Opioid & $70(46.7)$ & $59(84.3)$ & $11(15.7)$ & \\
\hline & Drug & $26(17.3)$ & $24(92.3)$ & $2(7.7)$ & \\
\hline & Inhalational & $9(6.0)$ & $8(88.9)$ & $1(11.1)$ & \\
\hline & Others & $19(12.7)$ & $16(84.2)$ & $3(15.8)$ & \\
\hline
\end{tabular}

Data are presented as Mean \pm SD or No. (\%)

LOS: Length of Stay; ICU: Intensive Care Unit; APACHE: Acute Physiology and Chronic Health Evaluation; SAPS: Simplified Acute Physiology Score; SOFA: Sequential Organ Failure Assessment 
Table 2. Accuracy of four prediction systems based on poisoning mechanisms in patients of poisoning ICU

\begin{tabular}{ccccc}
\hline Variables & Opioid & Drug & Inhalational & Others \\
\hline SOFA & $0.68(0.63-0.73)^{*}$ & $0.68(0.50-0.86)$ & $0.71(0.54-0.88)$ & $0.82(0.74-0.90)$ \\
SAPS II & $0.76(0.71-0.80)$ & $0.67(0.49-0.84)$ & $0.92(0.84-1.00)$ & $0.79(0.69-0.90)$ \\
APACHE II & $0.75(0.70-0.80)$ & $0.85(0.76-0.94)$ & $0.87(0.77-0.97)$ & $0.78(0.67-0.89)$ \\
APACHE IV & $0.77(0.72-0.82)$ & $0.89(0.83-0.96)$ & $0.70(0.56-0.84)$ & $0.71(0.58-0.83)$ \\
\hline
\end{tabular}

${ }^{*}$ Data are reported as the area under curve with $95 \%$ confidence interval.

International Journal of
Medical Toxicology \& Forensic Medicine

APACHE: Acute Physiology and Chronic Health Evaluation; SAPS: Simplified Acute Physiology Score; SOFA: Sequential Organ Failure Assessment

Considering the mechanism of poisoning, the most precise measure was APACHE IV (77.2\%) in opioid poisoning, APACHE IV (89.9\%) in drug poisoning, SAPS II $(92.5 \%)$ in inhalational poisoning, and SOFA (82.2\%) in other poisonings (Table 2).

As Table 3 shows, all four prediction systems are significantly correlated with each other. SAPS II has the highest correlation with GCS $(\mathrm{r}=-0.758, \mathrm{P}<0.001)$, and APACHE IV has the lowest but still significant relationship with GCS $(\mathrm{r}=-0.400, \mathrm{P}<0.001)$. Among these four systems, only APACHE IV $(\mathrm{r}=0.134, \mathrm{P}<0.001)$ and SAPS II $(\mathrm{r}=0.073, \mathrm{P}<0.05)$ have a significant correlation with the LOS in ICU.

Overall, all four systems had similar discriminatory power (i.e., the AUC) ranging in a narrow continuum from 0.72 to 0.77 . Similarly, the overall correct classification, which considers both survived and deceased ones ranged from $88.8 \%$ to $90.2 \%$. SOFA had the highest odds ratio for the prediction of mortality, and it had the highest calibration regarding the Hosmer-Lemeshow index (Table 4).

\section{Discussion}

In this study, we compared the ability of four ICU illness severity scoring systems (namely SOFA, SAPS II, APACHE II, and APACHE IV) to predict the prognosis of poisoned patients admitted to ICU. Our novelty in this study is that we evaluated the APACHE IV scoring system in the prediction of the mortality in poisoned patients in terms of various cofactors. Also, to the best of our knowledge, no study has compared the performance of such a potential prognostic system (APACHE IV) with older risk prediction systems (SOFA, SAPS II, and APACHE II) in mortality prediction in poisoned patients admitted to ICU.

Table 3. The correlation matrix for four prediction systems

\begin{tabular}{ccccccc}
\hline Variables & SOFA & SAPS II & APACHE II & APACHE IV & GCS & LOS in ICU \\
\hline SOFA & 1 & $0.183^{* *}$ & $0.175^{* *}$ & $0.147^{* *}$ & $-0.650^{* *}$ & -0.003 \\
SAPS II & $0.183^{* *}$ & 1 & $0.867^{* *}$ & $0.678^{* *}$ & $-0.758^{* *}$ & $0.073^{*}$ \\
APACHE II & $0.175^{* *}$ & $0.867^{* *}$ & 1 & $0.745^{* *}$ & $-0.708^{* *}$ & 0.050 \\
APACHE IV & $0.147^{* *}$ & $0.678^{* *}$ & $0.745^{* *}$ & 1 & $-0.400^{* *}$ & $0.134^{* *}$ \\
GCS & $-0.65^{* *}$ & $-0.758^{* *}$ & $-0.708^{* *}$ & $-0.400^{* *}$ & 1 & $-0.092^{*}$ \\
\hline LOS in ICU & -0.003 & $0.073^{*}$ & 0.050 & $0.134^{* *}$ & $-0.092^{*}$ & 1 \\
\hline
\end{tabular}

** $\mathrm{P}<0.001,{ }^{*} \mathrm{P}<0.05$. Full direct correlation is shown by red color $(\mathrm{r}=+1)$ and full reverse correlation is shown by blue color $(\mathrm{r}=-1)$.

SOFA: Sequential Organ Failure Assessment; SAPS: Simplified Acute Physiology Score; APACHE: Acute Physiology and Chronic Health Evaluation; GCS: Glasgow Coma Scale; LOS: Length of Stay; ICU: Intensive Care Unit.

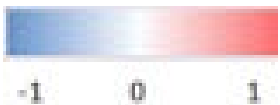


Table 4. Characteristics of various prediction systems for the first admission day in patients of poisoning ICU

\begin{tabular}{|c|c|c|c|c|c|}
\hline \multicolumn{2}{|c|}{ Charactristics } & \multirow{2}{*}{$\begin{array}{l}\text { SOFA } \\
1.32\end{array}$} & \multirow{2}{*}{$\begin{array}{c}\text { SAPS II } \\
1.06\end{array}$} & \multirow{2}{*}{$\begin{array}{c}\text { APACHE II } \\
1.15\end{array}$} & \multirow{2}{*}{$\begin{array}{c}\text { APACHE IV } \\
1.02\end{array}$} \\
\hline 10 - 1 i & Odds ratio & & & & \\
\hline & $95 \% \mathrm{Cl}$ & $1.08-1.62$ & $1.02-1.10$ & $1.06-1.24$ & $1.01-1.05$ \\
\hline \multirow{6}{*}{$\begin{array}{l}\text { Overall correct } \\
\text { classification, \% }\end{array}$} & Cox \& Snell R2 & 89.5 & 90.2 & 89.5 & 88.8 \\
\hline & Nagelkerke R2 & 0.05 & 0.09 & 0.09 & 0.04 \\
\hline & Hosmer-Lemeshow & 0.11 & 0.18 & 0.19 & 0.09 \\
\hline & $\chi^{2}$ & 4.98 & 13.09 & 12.83 & 9.73 \\
\hline & df & 8 & 8 & 8 & 8 \\
\hline & $P$ & 0.75 & 0.10 & 0.11 & 0.28 \\
\hline \multirow{5}{*}{$\begin{array}{c}\text { Receiver } \\
\text { operating } \\
\text { characteristic } \\
\text { analysis }\end{array}$} & Area under curve & 0.72 & 0.76 & 0.77 & 0.73 \\
\hline & $95 \% \mathrm{Cl}$ & $0.64-0.79$ & $0.68-0.83$ & $0.69-0.84$ & $0.65-0.80$ \\
\hline & Best cutoff & $>5$ & $>39$ & $>17$ & $>42$ \\
\hline & Sensitivity, \% & 80.0 & 86.6 & 80.0 & 86.6 \\
\hline & Specificity, \% & 59.6 & 72.0 & 68.2 & 52.7 \\
\hline
\end{tabular}

SOFA: Sequential Organ Failure Assessment; SAPS: Simplified Acute Physiology Score; APACHE: Acute Physiology and Chronic Health Evaluation

According to our results, based on Area Under The Receiver Operator Curve (AUROC) measures, the APACHE II risk prediction system has the best discriminative power (0.77) between survived and deceased patients with good sensitivity $(80 \%)$ and an acceptable specificity (68.2\%). Also, our study demonstrated that the SAPS II score (AUROC=0.76), APACHE IV score
(AUROC $=0.73$ ), and SOFA score (AUROC $=0.72$ ) systems had the best discriminative power compared with each other, after APACHE II scoring system. A commonly accepted approach expresses that an AUROC higher than 0.75 reflects strong and useful discrimination; 0.60 to 0.75 , possibly helpful discrimination; and less than 0.60 , poor discrimination [7]. According to this
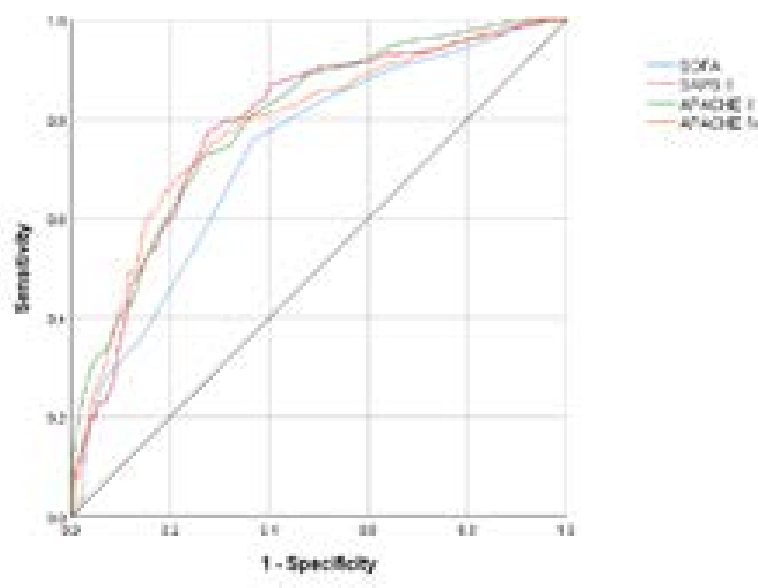

International Journal of
Medical Toxicology \& Forensic Medicine

Figure 1. The receiver operating characteristic curves for the four scoring systems under study

SOFA: Sequential Organ Failure Assessment; APACHE: Acute Physiology and Chronic Health Evaluation; SAPS: Simplified Acute Physiology Score. 


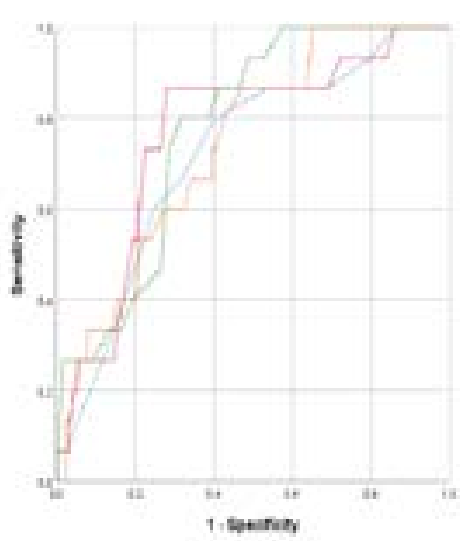

Day 1

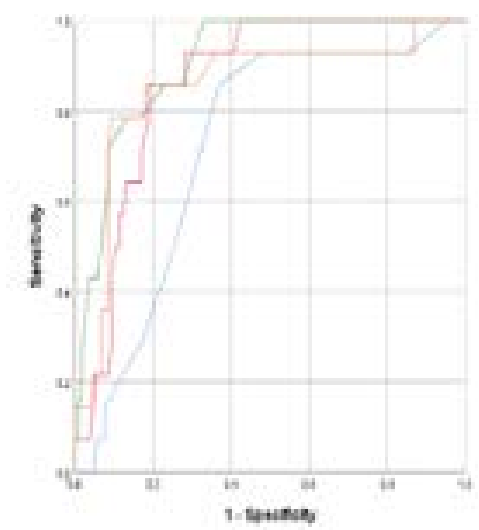

Day 3
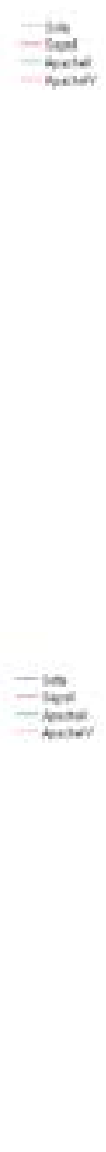

Day 5

Day 2
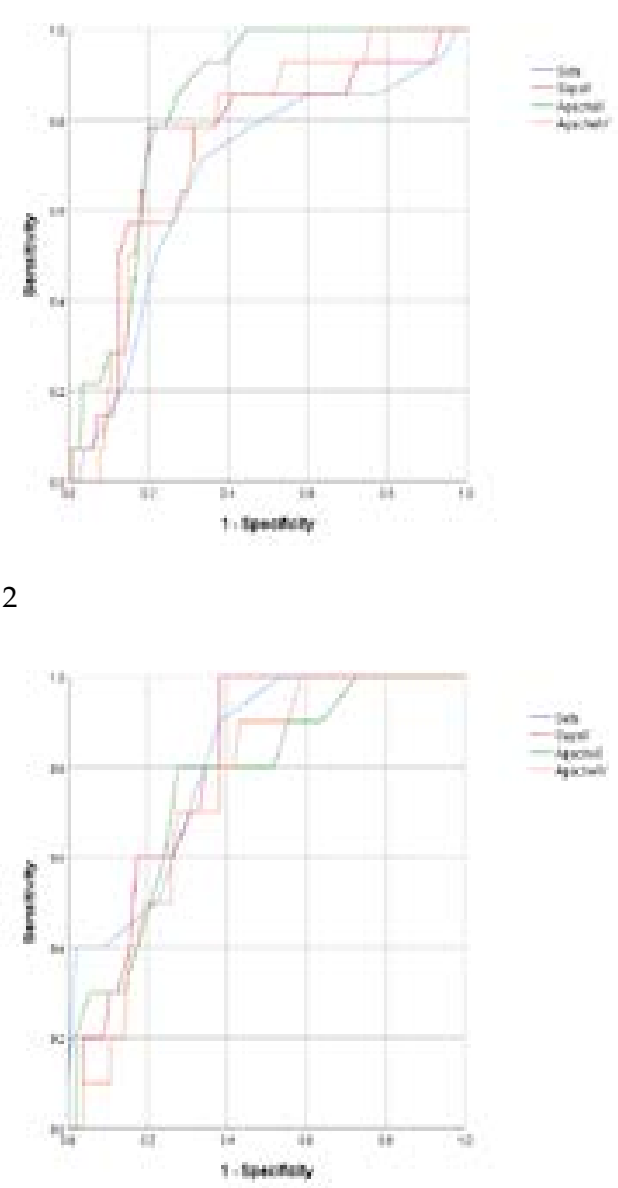

International Journal of
Medical Toxicology \& Forensic Medicine

Figure 2. Comparison of four prediction systems based on first to the fifth day of hospitalization in poisoning ICU

SOFA: Sequential Organ Failure Assessment; APACHE: Acute Physiology and Chronic Health Evaluation; SAPS: Simplified Acute Physiology Score.

approach, all four risk prediction systems had good discriminative power in poisoned patients.

Our results are compatible with the findings obtained by several previous reports [8-12]. The AUROC values in paraquat-poisoned patients and cardiac surgery patients for the APACHE II score system were 0.85 and 0.82 , for the SAPS II score system 0.83 and 0.80 , and for the SOFA score system 0.71 and $0.76[10,12]$. These findings confirm our results that APACHE II is better than SAPS II, and SAPS II is better than SOFA for mortality prediction in ICU patients.

In these studies and some other previous reports [11, 13], the SOFA score system had the lowest discriminatory power between survived and deceased patients as compared with the three other scoring systems. This result can be attributed to the design of the SOFA scoring system. It was primarily developed to describe the variations of severity during the organ failure process in ICU patients with sepsis [14]. Therefore, it is more suitable for morbidity prediction, although it can also be used for mortality prediction.

Among the poisoned patients, the most common indication for ICU admission is respiratory failure. This item is evaluated for all four systems (ventilator need, oxygen saturation, and oxygen therapy). Respiratory failure is also one of the causes of long-term ICU stay in patients with acute drug poisoning [15]. Besides, a considerable number of patients will lose consciousness following poisoning, which is another reason for ICU admission and or longer LOS.

The mean arterial pressure, serum $\mathrm{pH}$, and hematocrit are three items that are examined in both APACHE sys- 
tems but are not included in SOFA or SAPS II. Hypotension and acidosis (metabolic or respiratory) are common side effects of poisoning, especially in the end-stage phases. Hypotension is one of the predictors of mortality in patients with acute drug poisoning who were admitted to ICU [15]. The serum levels of albumin and glucose are only examined in APACHE IV.

On the other hand, only the SOFA system considered vasopressor administration. In SAPS II, systolic blood pressure is measured instead of mean arterial pressure. Age is not considered in SOFA, but it is present in the other three systems. Although poisoning is prevalent in younger individuals, older ones may need to be admitted to ICU for a longer period. Overall, the average age of poisoned patients admitted to ICU is lower than nonpoisoned ones admitted to $\operatorname{ICU}[16,17]$.

Serum sodium levels, heart rate, body temperature, and age are items that are not included in the SOFA system but all other three prediction systems. It seems that SAPS II is a weaker system compared to others in terms of evaluating renal function. Liisanantti and colleagues reported that renal failure is one of the factors relating to more prolonged ICU stay in patients with acute drug poisoning [15]. Our findings are congruent with previous results. SAPS II had the highest discriminative power for inhalational poisoning, which is more likely to lead to respiratory failure than renal failure.

Ratanarat et al. [8] and Alizadeh et al. [9] had compared APACHE II and SAPS II risk prediction systems in general- and poisoned-patients, respectively; and both of them showed that APACHE II was a better predictor of mortality based on AUROC measure. They had calculated the AUROC to be 0.78 for APACHE II, which are similar to our findings. In contrast to these studies, several studies reported that the SAPS II score was superior to the APACHE II score for mortality prediction $[13,18]$. Regardless of either APACHE II or SAPS II is better, many studies reported no statistically significant difference between APACHE II and SAPS II, and even a good correlation between them $[8,12,19-21]$. This conclusion was confirmed in our study, as the Pearson correlation test showed an excellent positive relationship between APACHE II and SAPS II ( $\mathrm{r}=0.87)$.

Although, according to our findings, APACHE IV score had a lower AUROC than APACHE II and SAPS II scoring systems, it had a higher AUROC than SOFA score. Similarly, Sun and colleagues demonstrated that SAPS II, APACHE IV, and SOFA had the best discriminatory power [11]. Also, Ibrahim and colleagues compared APACHE
IV and SAPS II in the mortality prediction of organophosphate-poisoned patients [22]. In contrast to our study, they showed that APACHE IV had better discriminatory power than SAPS II. It can be explained by the fact that Ibrahim's research was performed in organophosphate-poisoned patients. In contrast, our study has been conducted in all poisoned patients with various types of poisoning.

In addition to discrimination, calibration is another indicator that was used to evaluate the performance of risk prediction systems. All four scoring systems in this study indicated acceptable effectiveness when tested on calibration. However, the SOFA score indicated a little better efficiency than three other systems because of its higher $(\mathrm{P}>0.05)$ and lower Hosmer-Lemeshow value.

Our study was not without limitations. First, because the study was retrospective, all patient data could not be obtained. Therefore, incomplete data were expected. Second, we did not have monitored vital signs continuously, the existence of which could have provided more detailed data. However, it has been recommended to use the first available data for ICU admitted patients for practical reasons. The strength of this study is that four routine predictive systems were evaluated all together for an acceptable hospitalization period. To the best of our knowledge, this is the first report from the only poisoning ICU in the northeast of Iran.

We could conclude that the mortality of poisoned patients could be predicted using any of the four risk prediction systems upon ICU admission. Although all of these scoring systems can be used as a mortality predictor in poisoned patients in the ICU, the APACHE II score had strong discrimination power. So, we suggest that the APACHE II scoring system could be an acceptable tool in predicting the mortality of poisoned patients admitted to ICU.

\section{Ethical Considerations}

\section{Compliance with ethical guidelines}

The study protocol was approved by the Ethics Committee of Mashhad University of Medical Sciences (MUMS941481). Since medical confidentiality and personal data were preserved, the requirement for informed consent for this study from each patient or relatives was waived.

\section{Funding}

This research did not receive any specific grant from funding agencies in the public, commercial, or not-forprofit sectors. 


\section{Author's contributions}

Designing the study: Bita Dadpour, Alireza Sedaghat, Samaneh Silakhori, Farzad Mirzakhani; Supervision: Bita Dadpour; Data Collection: Samaneh Silakhori; Data analysis: Majid Khadem-Rezaiyan; Writing original draft: Majid Khadem-Rezaiyan, Bita Dadpour, Farzad Mirzakhani; Approving the final draft: Bita Dadpour, Majid Khadem-Rezaiyan, Farzad Mirzakhani.

\section{Conflict of interest}

The authors declared no conflict of interest.

\section{Acknowledgements}

We appreciate the support of the Clinical Research Development Unit of Akbar Hospital (affiliated to Mashhad University of Medical Sciences, Mashhad, Iran).

\section{References}

[1] Sawalha AF, Sweileh WM, Tufaha MT, Al-Jabi DY. Analysis of the pattern of acute poisoning in patients admitted to a governmental hospital in Palestine. Basic Clin Pharmacol Toxicol 2010; 107(5):914-8. [DOI:10.1111/j.1742-7843.2010.00601.x] [PMID]

[2] Gummin DD, Mowry JB, Spyker DA, Brooks DE, Fraser MO, Banner W. 2016 Annual report of the American Association of Poison Control Centers' National Poison Data System (NPDS): $34^{\text {th }}$ Ann report. Clin Toxicol (Phila). 2016; 55(10):1072254. [DOI:10.1080/15563650.2017.1388087] [PMID]

[3] Alinejad S, Zamani N, Abdollahi M, Mehrpour O. A narrative review of acute adult poisoning in Iran. Iran J Med Sci. 2017; 42(4):327. [PMCID]

[4] Nikfar S, Khatibi M, Abdollahiasl A, Abdollahi M. Cost and utilization study of antidotes: An Iranian experience. Int J Pharmacol. 2011; 7(1):46-9. [DOI: 10.3923/ijp.2011.46.49]

[5] Park SK, Chun HJ, Kim DW, Im TH, Hong HJ, Yi HJ. Acute physiology and chronic health evaluation ii and simplified acute physiology score ii in predicting hospital mortality of neurosurgical intensive care unit patients. J Korean Med Sci. 2009; 24(3):420-6. [DOI:10.3346/jkms.2009.24.3.420] [PMID] [PMCID]

[6] Sakr Y, Krauss C, Amaral AC, Réa-Neto A, Specht M, Reinhart K, Marx G. Comparison of the performance of SAPS II, SAPS 3, APACHE II, and their customized prognostic models in a surgical intensive care unit. Br J Anaesth. 2008; 101(6):798803. [DOI:10.1093/bja/aen291] [PMID]

[7] Hosmer DW, Lemeshow S. Assessing the Fit of the Model. In: Hosmer DW, Lemeshow S, editors. Applied Logistic Regression. $2^{\text {nd }}$ ed. New York: John Wiley \& Sons; 2000. [DOI:10.1002/0471722146.ch5]
[8] Ratanarat R, Thanakittiwirun M, Vilaichone W, Thongyoo $S$, Permpikul C. Prediction of mortality by using the standard scoring systems in a medical intensive care unit in Thailand. J Med Assoc Thai. 2005; 88(7):949-55. [PMID]

[9] Alizadeh AM, Hassanian-Moghaddam H, Shadnia S, Zamani N, Mehrpour O. Simplified acute physiology score II/ acute physiology and chronic health evaluation II and prediction of the mortality and later development of complications in poisoned patients admitted to intensive care unit. Basic Clin Pharmacol Toxicol. 2014; 115(3):297-300. [DOI:10.1111/ bcpt.12210] [PMID]

[10] Lee JH, Hwang SY, Kim HR, Kim YW, Kang MJ, Cho KW, et al. Effectiveness of the sequential organ failure assessment, acute physiology and chronic health evaluation II, and simplified acute physiology score II prognostic scoring systems in paraquat-poisoned patients in the intensive care unit. Hum Exp Toxicol. 2017; 36(5):431-7. [DOI:10.1177/0960327116657602] [PMID]

[11] Sun D, Ding H, Zhao C, Li Y, Wang J, Yan J, et al. Value of SOFA, APACHE IV and SAPS II scoring systems in predicting short-term mortality in patients with acute myocarditis. Oncotarget. 2017; 8(38):63073-83. [DOI:10.18632/oncotarget.18634] [PMID] [PMCID]

[12] Exarchopoulos T, Charitidou E, Dedeilias P, Charitos C, Routsi C. Scoring systems for outcome prediction in a cardiac surgical intensive care unit: A comparative study. Am J Crit Care. 2015; 24(4):327-34. [DOI:10.4037/ajcc2015500] [PMID]

[13] Sharma S, Gupta A, Virmani SK, Lal R. Assessment and comparison of 3 mortality prediction models SAPS II, APACHE II and SOFA for prediction of mortality in patients of sepsis. Int J Adv Med. 2017; 4(3):623-9. [DOI:10.18203/23493933.ijam20171476]

[14] Vincent JL, Moreno R, Takala J, Willatts S, De Mendonca A, Bruining H, et al. The SOFA (Sepsis-related Organ Failure Assessment) score to describe organ dysfunction/failure. On behalf of the working group on sepsis-related problems of the European society of intensive care medicine. Intensive Care Med. 1996; 22(7):707-10. [DOI:10.1007/bf01709751] [PMID]

[15] Liisanantti JH, Ohtonen P, Kiviniemi O, Laurila JJ, AlaKokko TI. Risk factors for prolonged intensive care unit stay and hospital mortality in acute drug-poisoned patients: an evaluation of the physiologic and laboratory parameters on admission. J Crit Care. 2011; 26(2):160-5. [DOI:10.1016/j. jcrc.2010.08.009] [PMID]

[16] Shadnia S, Zamani N, Hassanian-Moghaddam H, Shafaroodi $\mathrm{H}$, Padandar M, Rezaeizadeh MH. Prognostic value of cortisol and thyroid function tests in poisoned patients admitted to toxicology ICU. World J Emerg Med. 2018; 9(1):51-5 [DOI:10.5847/wjem.j.1920-8642.2018.01.008] [PMID] [PMCID]

[17] Khodabandeh F, Shadnia S, Pormatine M. Performance assessment of the acute physiology and chronic health evaluation iv scoring system in outcome of patients fallowing acute poisoning. Novelty Biomed. 2018; 6(4):174-9. [DOI: 10.22037/nbm.v6i4.21277]

[18] Sungurtekin H, Gurses E, Balci C. Evaluation of several clinical scoring tools in organophosphate poisoned patients. Clin Toxicol (Phila). 2006; 44(2):121-6. [DOI:10.1080/15563650500514350] [PMID]

[19] Kim YH, Yeo JH, Kang MJ, Lee JH, Cho KW, Hwang S, et al. Performance assessment of the SOFA, APACHE II scoring system, and SAPS II in intensive care unit organophosphate 
poisoned patients. J Korean Med Sci. 2013; 28(12):1822-6.

[DOI:10.3346/jkms.2013.28.12.1822] [PMID] [PMCID]

[20] Godinjak A, Iglica A, Rama A, Tancica I, Jusufovic S, Ajanovic A, et al. Predictive value of SAPS II and APACHE II scoring systems for patient outcome in a medical intensive care unit. Acta Medica Academica. 2016; 45(2):97-103. [DOI:10.5644/ama2006-124.165]

[21] Fadaizadeh L, Tamadon R, Saeedfar K, Jamaati HR. Performance assessment of acute physiology and chronic health evaluation ii and simplified acute physiology score ii in a referral respiratory intensive care unit in Iran. Acta Anaesthesiol Taiwan. 2012; 50(2):59-62. [DOI:10.1016/j.aat.2012.05.004] [PMID]

[22] Ibrahim MA, El Masry MK, Moustafa AA, Hagras AM, Ali NM. Comparison of the accuracy of two scoring systems in predicting the outcome of organophosphate intoxicated patients admitted to Intensive Care Unit (ICU). Egypt J Forensic Sci. 2011; 1(1):41-7. [DOI:10.1016/j.ejfs.2011.04.013] 
This Page Intentionally Left Blank 